\title{
A Note on an Expansion of Hypergeometric Functions of Two Variables
}

\author{
By Arun Verma
}

1. In 1927 Fox [5] obtained the expansions of a product of two Bessel functions in a series of product of a Bessel function and a Gaussian hypergeometric function. Later on Rice [8] and Bailey [2] discovered a number of results of this type, some of them contained the result of Fox as a particular case.

Recently, Srivastava [9] gave four general expansions of products of generalised hypergeometric functions in a series of product of generalised hypergeometric functions of two variables and a Gaussian hypergeometric function, which incorporated as a special case the results of Fox, Rice and Bailey mentioned above.

The aim of the present note is to derive an expansion of a generalised hypergeometric function of two variables in a series of product of generalised hypergeometric functions of two variables and a generalised hypergeometric function. The result deduced is further generalised to an expansion of a Meijer's $G$-function of two variables (defined recently by Agarwal) in a series of product of a Meijer's G-function and a hypergeometric function of two variables.

The results obtained are very general and contain as special cases the expansions of Jerry L. Fields and Jet Wimp [6], L. Carlitz and W. Alsalam [4], Meijer [7] and many other results. The results are obtained by the use of the Laplace transform and the inverse Laplace transform as has been done by the author elsewhere also [11], [12].

The following notation due to Chaundy [3] shall be used to represent the hypergeometric function of higher order and of two variables

$$
F\left[\begin{array}{l}
\left(a_{p}\right):\left(b_{q}\right) ;\left(r_{s}\right) ; \\
\left(A_{P}\right):\left(B_{Q}\right) ;\left(R_{S}\right)
\end{array} x, y\right]=\sum_{m=0}^{\infty} \sum_{n=0}^{\infty} \frac{\left[\left(a_{p}\right)\right]_{m+n}\left[\left(b_{q}\right)\right]_{m}\left[\left(r_{s}\right)\right]_{n}}{[1]_{m}[1]_{n}\left[\left(A_{P}\right)\right]_{m_{s}+n}\left[\left(B_{Q}\right)\right]_{m}\left[\left(R_{S}\right)\right]_{n}} x^{m} y^{n},
$$

where $\left(\alpha_{m, n}\right)$ shall mean $[n-m+1]$ parameters $\alpha_{m}, \alpha_{m+1}, \cdots \alpha_{n}$. But when $m=1$, we shall write $\left(\alpha_{n}\right)$ instead of $\left(\alpha_{1, n}\right)$. Also as usual $\Gamma\left[\left(\alpha_{p}\right) ;\left(\beta_{q}\right)\right]$ denotes $\left\{\prod_{j=1}^{p} \Gamma\left[\alpha_{j}\right]\right\}\left\{\prod_{j=1}^{q} \Gamma\left[\beta_{j}\right]\right\}^{-1}$.

2. The result to be deduced is:

$$
\begin{aligned}
F\left[\begin{array}{l}
\left(e_{r}\right),\left(f_{R}\right):\left(a_{p}\right),\left(c_{P}\right) ; \\
\left(g_{s}\right),\left(h_{S}\right):\left(b_{q}\right) ;\left(d_{Q}\right)
\end{array} x, y z\right] \\
\quad=\sum_{n=0}^{\infty} \frac{[2 \lambda]_{n}\left[\left(e_{r}\right)\right]_{n}}{[1]_{n}[\lambda]_{n}\left[\left(g_{s}\right)\right]_{n}}(-z / 4)_{r+1}^{n} F_{s+1}\left[\begin{array}{l}
\lambda+n+\frac{1}{2},\left(e_{r}\right)+n ; z \\
2 \lambda+2 n+1,\left(g_{s}\right)+n
\end{array}\right] \\
\quad \times F\left[\begin{array}{l}
-n, 2 \lambda+n,\left(f_{R}\right):\left(a_{p}\right) ;\left(c_{P}\right) ; x, y \\
\lambda+\frac{1}{2},\left(h_{S}\right):\left(b_{q}\right) ;\left(d_{Q}\right)
\end{array}\right]
\end{aligned}
$$

provided $r+R+p<1+s+S+q, r+R+P<1+s+S+Q, r<s+1$ or $r=s+1$ and $|z|<1$ and the series on the right-hand side has a meaning.

To prove (2.1) we start from the result of Srivastava [9]

Received October 13, 1965. 


$$
\begin{aligned}
\left(\frac{1}{2} z\right)^{\lambda}{ }_{p} F_{q}\left[\begin{array}{l}
\left(a_{p}\right) ; x z \\
\left(b_{q}\right)
\end{array}\right]{ }_{P} F_{Q}\left[\begin{array}{l}
\left(c_{P}\right) ; y z \\
\left(d_{Q}\right)
\end{array}\right] \\
=\frac{\Gamma[\lambda]}{\Gamma[2 \lambda]} e^{z} \sum_{n=0}^{\infty}(-)^{n} \frac{[\lambda+n] \Gamma[2 \lambda+n]}{[1]_{n}} I_{\lambda+n}(z) \\
\times F\left[\begin{array}{l}
\left.-n, 2 \lambda+n:\left(a_{p}\right) ;\left(c_{P}\right) ; \frac{1}{2} x, \frac{1}{2} y\right] \\
\lambda+\frac{1}{2}:\left(b_{q}\right) ;\left(d_{Q}\right)
\end{array}\right.
\end{aligned}
$$

But we know that

$$
I_{\lambda}\left(\frac{1}{2} x\right)=(x / 4)^{\lambda} e^{-x / 2} F_{1}\left[\lambda+\frac{1}{2} ; 2 \lambda+1 ; x\right] .
$$

Substituting (2.3) in (2.2) and after a little simplification we get that

$$
\begin{array}{r}
F\left[\begin{array}{r}
:\left(a_{p}\right) ;\left(c_{P}\right) ; \\
:\left(b_{q}\right) ;\left(d_{Q}\right)
\end{array} x z, y z\right]=\sum_{n=0}^{\infty} \frac{[2 \lambda]_{n}(-z / 4)^{n}}{[1]_{n}[\lambda]_{n}}{ }_{1} F_{1}\left[\begin{array}{l}
\lambda+n+1 ; z \\
2 \lambda+2 n+1
\end{array}\right] \\
\times F\left[\begin{array}{c}
-n, 2 \lambda+n:\left(a_{p}\right) ;\left(c_{P}\right) ; x, y \\
\lambda+\frac{1}{2}:\left(b_{q}\right) ;\left(d_{Q}\right)
\end{array}\right) .
\end{array}
$$

Then we deduce (2.1) by the method of finite mathematical induction by using the Laplace transform and the inverse Laplace transform. To do so, assume that the result holds for some values of $p, P, q, Q, r, R, s$ and $S$, then replace $z$ by $z t$ on both the sides, multiply by $t^{e_{r+1}-1}$ and take the Laplace transform with respect to " $t$ ". Then using the result

$$
\Gamma[z]=\int_{0}^{\infty} e^{-t} t^{z-1} d t
$$

we find that the $r$ is replaced by $[r+1]$. This completes the induction with respect to $r$. To effect the induction with respect to $s$ replace $z$ by $z / t$ and multiply both sides by $t^{-g_{8+1}}$ and take the inverse Laplace transform with respect to " $t$ " and then using the result

$$
\frac{1}{\Gamma[z]}=\frac{1}{2 \pi i} \int_{c-i \infty}^{c+i \infty} e^{t} t^{-z} d t
$$

we find that the $s$ is replaced by $[s+1]$. This completes the induction with respect to $s$. Similarly the induction with respect to $R$ and $S$ can be effected. But for $r=s=R=S=0$, the result (2.1) reduces to (2.4) and thus the proof of (2.1) by induction is complete.

The result (2.1) is very general in nature and contains as a special case the results of Fox [5], Rice [8], Bailey [2], Jerry L. Fields and Jet Wimp [6] and Srivastava [9].

3. In this section we intend to generalise the result further to an expansion involving a certain number of "free" parameters. To do so we proceed as follows.

Replace $s$ by $s+1$ and $R$ by $R+1$ and set $g_{s+1}=\lambda+\frac{1}{2}, f_{R+1}=\lambda+\frac{1}{2}$ and then putting $x=0=y$, and replacing $2 \lambda$ by $\lambda,(2.1)$ becomes

$$
1=\sum_{n=0}^{\infty} \frac{\left[\left(e_{r}\right)\right]_{n}(-z)^{n}}{[1]_{n}[\lambda+n]_{n}\left[\left(g_{s}\right)\right]_{n}}{ }_{r} F_{s+1}\left[\begin{array}{l}
\left(e_{r}\right)+n ; \\
\left(g_{s}\right)+n, \lambda+2 n+1
\end{array}\right] .
$$

Then replacing $\left(e_{r}\right),\left(g_{s}\right)$ and $\lambda$ by $\left(e_{r}\right)+k+K,\left(g_{s}\right)+k+K$ and $\lambda+2 k+2 K$ respectively, multiplying both sides by 


$$
\frac{\left[\left(g_{s}\right)\right]_{k+K}\left[\left(a_{p}\right)\right]_{k}\left[\left(b_{P}\right)\right]_{K}}{[1]_{k}[1]_{K}\left[\left(h_{G}\right)\right]_{k+K}\left[\left(c_{d}\right)\right]_{k}\left[\left(e_{D}^{\prime}\right)\right]_{K}}(x z)^{k}(y z)^{K}
$$

and summing both sides with respect to $k$ and $K$ from 0 to $\infty$, and lastly setting $k+n+t=n$, changing the order of summation so that the summation with respect to " $t$ " is performed last, we get that

$$
\begin{aligned}
& F\left[\begin{array}{l}
\left(f_{o}\right):\left(a_{p}\right) ;\left(b_{P}\right) ; x z, y z \\
\left(h_{G}\right):\left(c_{d}\right) ;\left(e_{D}{ }^{\prime}\right)
\end{array}\right]=\sum_{t=0}^{\infty} \frac{\left[\left(e_{r}\right)\right]_{t}(-z)^{t}}{[1]_{t}\left[\left(g_{s}\right)\right]_{t}[\lambda+t]_{t}} \\
& \times{ }_{r} F_{s+1}\left[\begin{array}{l}
\left(e_{r}\right)+t ; z \\
\left(g_{s}\right)+t, \lambda+2 t+1
\end{array}\right] F\left[\begin{array}{l}
\left.\left(g_{s}\right),\left(f_{\theta}\right), \lambda+t,-t:\left(a_{p}\right) ;\left(b_{P}\right) ; x, y\right] \\
\left(e_{r}\right),\left(h_{G}\right):\left(c_{d}\right) ;\left(e_{D}{ }^{\prime}\right)
\end{array}\right.
\end{aligned}
$$

The result (3.1) can then be generalised by using the Laplace and the inverse Laplace transform as in $\$ 2$ to the form

$$
\begin{aligned}
& F\left[\begin{array}{l}
\left(f_{g}\right),\left(\alpha_{m}\right):\left(a_{p}\right) ;\left(b_{P}\right) ; \\
\left(h_{G}\right),\left(\beta_{n}\right):\left(c_{d}\right) ;\left(e_{D}^{\prime}\right)
\end{array} x z, y z\right]=\sum_{t=0}^{\infty} \frac{\left[\left(e_{r}\right)\right]_{t}\left[\left(\alpha_{m}\right)\right]_{t}(-z)^{t}}{[1]_{t}\left[\left(g_{s}\right)\right]_{t}\left[\left(\beta_{n}\right)\right]_{t}[\lambda+t]_{t}} \\
& \times_{r+m} F_{s+n+1}\left[\begin{array}{l}
\left(e_{r}\right)+t,\left(\alpha_{m}\right)+t ; z \\
\left(\beta_{n}\right)+t,\left(g_{s}\right)+t, \lambda+2 t+1
\end{array}\right] \\
& \times F\left[\begin{array}{l}
\left.\left(g_{s}\right),\left(f_{g}\right), \lambda+t,-t:\left(a_{p}\right) ;\left(b_{P}\right) ; x, y\right] \\
\left(e_{r}\right),\left(h_{G}\right):\left(c_{d}\right) ;\left(e_{D}^{\prime}\right)
\end{array}\right],
\end{aligned}
$$

provided $g+m+p<1+G+n+d, g+m+P<1+G+n+D, r+m<$ $s+n$ or $r+m=s+n$ and $|z|<1$ and the series on the right-hand side converges.

This result contains as a special case the result deduced in the previous section and also incorporates a result due to Jerry L. Fields and Jet Wimp, which in turn incorporates the results of Chaundy [3], Carlitz and Alsalam [4] and Meijer [7] as special cases.

4. In this section we intend to generalise the result (3.1) to a result giving the expansion of Meijer's $G$-function of two variable, defined recently by Agarwal [1]* . Agarwal has defined the $G$-function of two variable as

$$
G_{p,\left[t: t^{\prime}\right], s,\left[q: q^{\prime}\right]}^{n, \nu_{1}, \nu_{2}, m_{1}, m_{2}}\left[\begin{array}{l}
x \\
y \\
\left(\gamma_{t}\right) ;\left(\gamma_{t^{\prime}}^{\prime}\right) \\
\left(\delta_{s}\right) \\
\left(\beta_{q}\right) ;\left(\beta_{q^{\prime}}^{\prime}\right)
\end{array}\right]=-\frac{1}{4 \pi^{2}} \int_{-i \infty}^{i \infty} \int_{-i \infty}^{i \infty} \Phi(\xi+\eta) \psi(\xi, \eta) x^{\xi} y^{\eta} d \xi d \eta
$$

where

$\psi(\xi, \eta)=\Gamma\left[\begin{array}{c}\left(\beta_{m_{1}}\right)-\xi,\left(\gamma_{\nu_{1}}\right)+\xi,\left(\beta_{m_{2}}^{\prime}\right)-\eta,\left(\gamma_{\nu_{2}}^{\prime}\right)+\eta \\ 1-\left(\beta_{m_{1}+1, q}\right)+\xi, 1-\left(\gamma_{\nu_{1}+1, t}\right)-\xi, 1-\left(\beta_{m_{2}+1, q^{\prime}}^{\prime}\right)+\eta, \\ 1-\left(\gamma_{\nu_{2}+1, t^{\prime}}^{\prime}\right)-\eta\end{array}\right]$,

$\Phi(\xi+\eta)=\Gamma\left[\begin{array}{l}1-\left(\epsilon_{n}\right)+\xi+\eta \\ \left(\epsilon_{n+1, p}\right)-\xi-\eta,\left(\delta_{s}\right)+\xi+\eta\end{array}\right]$

where $0 \leqq m_{1} \leqq q, 0 \leqq m_{2} \leqq q^{\prime}, 0 \leqq \nu_{1} \leqq t, 0 \leqq \nu_{2} \leqq t^{\prime}, 0 \leqq n \leqq p$.

* I am indebted to Professor R. P. Agarwal who has so kindly allowed me to go through his unpublished manuscript [1]. It may be mentioned that the $G$-function of two variable which we are taking is a slight variant of the one given by Agarwal, though in essence the function is the same. 
The sequence of parameters $\left(\beta_{m_{1}}\right),\left(\beta_{m_{2}}^{\prime}\right),\left(\gamma_{\nu_{1}}\right),\left(\gamma_{\nu_{2}}^{\prime}\right)$ and $\left(\epsilon_{n}\right)$ are such that none of the poles of the integrand coincide. The paths of integration are indented, if necessary, in such a manner that all the poles of $\Gamma\left[\beta_{j}-\xi\right], j=1,2, \cdots, m_{1}$, and $\Gamma\left[\beta_{k}{ }^{\prime}-\eta\right], k=1,2, \cdots, m_{2}$ lie to the right and those of $\Gamma\left[\gamma_{j}+\xi\right], j=1,2, \cdots, \nu_{1}$ and $\Gamma\left[\gamma_{k}{ }^{\prime}+\eta\right], k=1,2, \cdots, \nu_{2}$ and $\Gamma\left[1-\epsilon_{j}+\xi+\eta\right], j=1,2, \cdots, n$ lie to the left of the imaginary axis.

The integral (4.1) converges if

$$
\begin{aligned}
p+q+s+t & <2\left(m_{1}+\nu_{1}+n\right), \\
p+q^{\prime}+s+t^{\prime} & <2\left(m_{2}+\nu_{2}+n\right),
\end{aligned}
$$

and

$$
\begin{aligned}
& |\arg x|<\pi\left[m_{1}+\nu_{1}+n-\frac{1}{2}(p+q+s+t)\right], \\
& |\arg y|<\pi\left[m_{2}+\nu_{2}+n-\frac{1}{2}\left(p+q^{\prime}+s+t^{\prime}\right)\right]
\end{aligned}
$$

Then using $[1 ; 3.3]$, we can write $(3.2)$ in the form $(x z)^{-c_{1}}(y z)^{-e_{1}} G_{g+m,[p: P], G+n,[1+d: 1+D]}^{g+m, p, P, 1,1}$

$$
\begin{aligned}
& {\left[\begin{array}{l|l}
-x z & \begin{array}{l}
1-\left(f_{g}\right)+c_{1}+e_{1}^{\prime}, 1-\left(\alpha_{m}\right)+c_{1}+e_{1}^{\prime} \\
\left(a_{p}\right)-c_{1} ;\left(b_{P}\right)-e_{1}^{\prime} \\
\left(h_{G}\right)-c_{1}-e_{1}^{\prime},\left(\beta_{n}\right)-c_{1}-e_{1}^{\prime} \\
1-c_{1}, 2-\left(c_{d}\right)-c_{1} ; 1-e_{1}^{\prime}, 2-\left(e_{D}^{\prime}\right)-e_{1}^{\prime}
\end{array}
\end{array}\right]} \\
& =\Gamma\left[\begin{array}{l}
\left(a_{p}\right)-c_{1},\left(b_{P}\right)-e_{1}^{\prime}, 1-\left(f_{g}\right)+c_{1}+e_{1}^{\prime}, 1-\left(\alpha_{m}\right)+c_{1}+e_{1}^{\prime},\left(g_{s}\right),\left(\beta_{n}\right) ; \\
\left(h_{G}\right)-c_{1}-e_{1}^{\prime},\left(\beta_{n}\right)-c_{1}-e_{1}^{\prime}, 2-\left(c_{d}\right)-c_{1}, 2-\left(e_{D}^{\prime}\right)-e_{1}^{\prime},\left(e_{r}\right),\left(\alpha_{m}\right)
\end{array}\right] \\
& \times \sum_{t=0}^{\infty} \frac{(-z)^{t}}{[1]_{t}[\lambda+t]_{t}} F\left[\begin{array}{l}
\left.\left(g_{s}\right),\left(f_{g}\right), \lambda+t,-t:\left(a_{p}\right) ;\left(b_{P}\right) ; x, y\right] \\
\left(e_{r}\right),\left(h_{G}\right):\left(c_{d}\right) ;\left(e_{D}^{\prime}\right)
\end{array}\right] \\
& \times G_{1+n+s, m+r}^{m+r, 1}\left(\begin{array}{l|l}
-1 / z & \begin{array}{l}
1,\left(\beta_{n}\right)+t,\left(g_{s}\right)+t \\
\left(\alpha_{m}\right)+t,\left(e_{r}\right)+t
\end{array}
\end{array}\right) .
\end{aligned}
$$

Then applying the Laplace transform and its inverse this relation can be generalised to the form

$(x z)^{-c_{1}}(y z)^{-e_{1}{ }^{\prime}} G_{g+m+N,[p+v: V+P], G+n,[1+d+l: 1+D+L]}^{g+m, p, l, L}$

$$
\left[\begin{array}{l|l}
-x z & \begin{array}{l}
1-\left(f_{g}\right)+c_{1}+e_{1}^{\prime}, 1-\left(\alpha_{m}\right)+c_{1}+e_{1}^{\prime},\left(\gamma_{N}\right)+c_{1}+e_{1}^{\prime} \\
\left(a_{p}\right)-c_{1},\left(\chi_{v}\right)-c_{1} ;\left(b_{P}\right)-c_{1},\left(x_{v}^{\prime}\right)-e_{1}^{\prime} \\
\left(h_{G}\right)-c_{1}-e_{1}^{\prime},\left(\beta_{n}\right)-c_{1}-e_{1}^{\prime} \\
1-c_{1}, 2-\left(c_{d}\right)-c_{1},\left(\delta_{l}\right)-c_{1} ; 1-e_{1}^{\prime}, 2-\left(e_{D}^{\prime}\right)-e_{1}^{\prime},\left(\epsilon_{L}\right)-e_{1}^{\prime}
\end{array}
\end{array}\right]
$$

$$
\begin{aligned}
=\Gamma & {\left[\begin{array}{r}
\left(a_{p}\right)-c_{1},\left(b_{P}\right)-e_{1}^{\prime}, 1-\left(f_{g}\right)+c_{1}+e_{1}^{\prime}, 1-\left(\alpha_{m}\right) \\
+c_{1}+e_{1}^{\prime},\left(g_{s}\right),\left(\beta_{n}\right),\left(\delta_{l}\right),\left(\epsilon_{L}\right) ; \\
\left(h_{G}\right)-c_{1}-e_{1}^{\prime},\left(\beta_{n}\right)-c_{1}-e_{1}^{\prime}, 2-\left(c_{d}\right)-c_{1}, \\
2-\left(e_{D}^{\prime}\right)-e_{1}^{\prime},\left(e_{r}\right),\left(\alpha_{m}\right), 1-\left(\chi_{v}\right), 1-\left(\chi_{v}{ }^{\prime}\right)
\end{array}\right] } \\
& \left.\times \sum_{t=0}^{\infty} \frac{(-z)^{t}}{[1]_{t}[\lambda+t]_{t}} G_{1+n+s, m+r+N}^{m+r, 1}-1 / z \mid \begin{array}{l}
1,\left(\beta_{n}\right)+t,\left(g_{s}\right)+t \\
\left(\alpha_{m}\right)+t,\left(e_{r}\right)+t,\left(\gamma_{N}\right)+t
\end{array}\right) \\
& \times F\left[\begin{array}{l}
\left(g_{s}\right),\left(f_{g}\right), \lambda+t,-t:\left(\chi_{v}\right),\left(\delta_{l}\right),\left(a_{p}\right) ;\left(\chi_{v}{ }^{\prime}\right),\left(\epsilon_{L}\right),\left(b_{P}\right) ; x, y \\
\left(e_{r}\right),\left(h_{G}\right):\left(c_{d}\right),\left(e_{D}^{\prime}\right)
\end{array}\right] .
\end{aligned}
$$


It may be noted that though (4.2) does not directly incorporates the results of Wimp and Luke [13] and Meijer [7], yet they could be deduced easily by using the Laplace transform technique to its relevant special cases. It does not appear to be possible to give a direct generalisation of the above results in the form (4.2).

The basic analogue of (3.2) can also be obtained by starting from a known result due to Verma [11] and follow exactly the method described in $\$ 3$.

Department of Mathematics,

The University, Gorakhpur,

India.

1. R. P. Agarwal, "An extension of Meijer's G-function," Proc. Nat. Inst. Sci. India Part A, v. 32,1965 . (To appear.)

2. W. N. BAILEY, "Some expansions in Bessel functions involving Appell functions $F_{4}$," Quart. J. Math. Oxford Ser., v. 6, 1935, pp. 233-238.

3. T. W. Chaundy, "Expansions of hypergeometric functions," Quart. J. Math. Oxford Ser., v. 13, 1942, pp. 159-171 MR 4, 197.

4. L. CARLitz \& W. A. Al-Salam, "Some functions associated with Bessel functions," $J$. Math. Mech., v. 12, 1963, pp. 911-933. MR 27 * 4962.

5. C. Fox, "The expansion of hypergeometric function in terms of similar series," Proc. London Math. Soc., v. 26, 1927, pp. 201-210.

6. J. L. FIELDS \& JET WIMP, "Expansions of hypergeometric functions in hypergeometric functions," Math. Comp., v. 15, 1961, pp. 390-396. MR 23 * A3289.

7. C.'S. MeiJer, "Expansion theorems for the $G$-function,"

a) I, II, Nederl. Akad. Wetensch. Proc. Ser. A, v. $55=$ Indag. Math., v. 14, 1952, pp. 369$379,483-487$. MR 14, 469, 642;

b) III, IV, V, Nederl. Akad. Wetensch. Proc. Ser. A, v. $56=$ Indag. Math., v. 15, 1953, pp. 43-49, 187-193, 349-357. MR 14, 748, 979; MR 15, 422;

c) ibid., VI, VII, VIII, Nederl. Akad. Wetensch. Proc. Ser. A, v. $57=$ Indag. Math., v. 16, 1954, pp. 77-82, 83-91, 273-279. MR 15, 791, 955;

d) ibid., IX, X, Nederl. Akad. Wetensch. Proc. Ser. A, v. 58 = Indag. Math., v. 17, 1955, pp. 243-251, 309-314. MR 16, 1106;

e) ibid., XI, Nederl. Akad. Wetensch. Proc. Ser. A, v. 59 = Indag. Math., v. 18, 1956, pp. 70-82. MR 17, 846.

8. S. O. Rice, "On contour integrals for the product of Bessel functions," Quart. J. Math. Oxford Ser., v. 6, 1935, pp. 52-64.

9. H. M. Sirivastava, "Some expansions in product of hypergeometric functions," Proc. Cambridge Philos. Soc., v. 62, 1965.

10 . A. Verma, "A class of expansions of $G$-functions and the Laplace transform," Math. Comp., v. 19, 1965 , pp. 661-664.

11. A. Verma, "Certain expansions involving generalised basic hypergeometric series," Math. Comp., v. 20, 1966, pp. 151-157.

12. A. Verma, "Expansions of hypergeometric functions of two variables," Collect. Math. (To appear.)

13. JET WIMP \& Y. L. LUKE, "Expansion formulas for generalised hypergeometric functions," Rend. Circ. Mat. Palermo, (2), v. 11, 1962, pp. 351-366. 